\title{
Effect of yogic intervention on the autonomic nervous system in the patients with coronary artery disease: a randomized controlled trial
}

\author{
A. Pal, ${ }^{1}$ N. Srivastava, ${ }^{1}$ V.S. Narain, ${ }^{2}$ G.G. Agrawal ${ }^{3}$ and M. Rani ${ }^{4}$
}

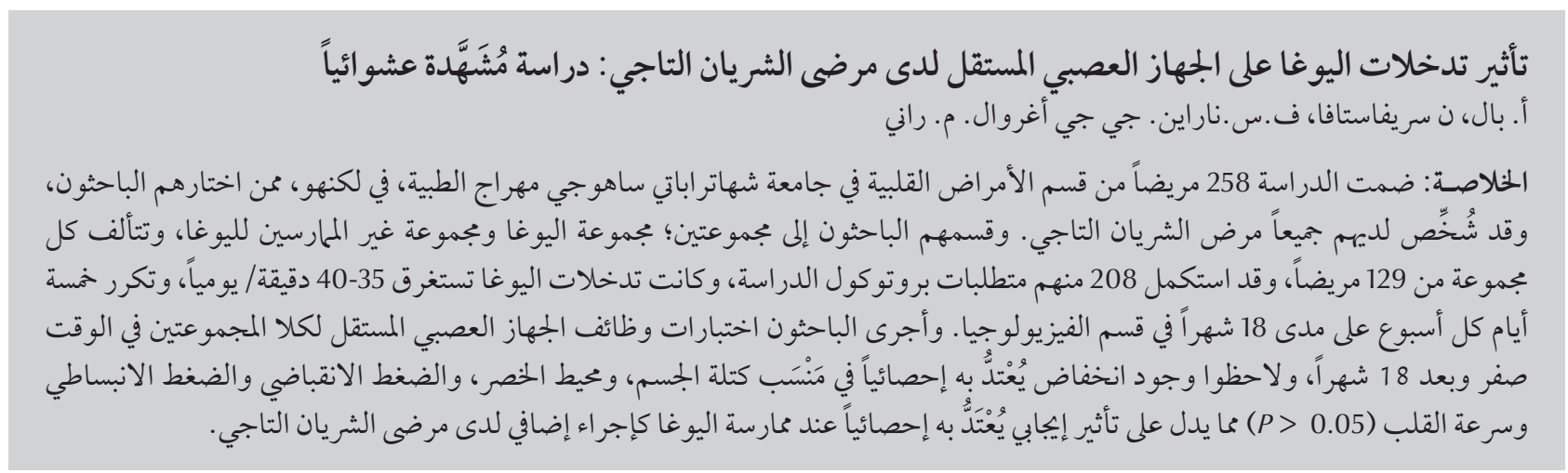

ABSTRACT In this study 258 patients from the Department of Cardiology in Chhatrapati Shahuji Maharaj Medical University, Lucknow were selected to participate. All had been diagnosed with coronary artery disease. They were randomly divided into 2 groups, the yoga group and the non-yoga group, 129 in each group; 208 completed the study protocol. The yogic intervention consisted of 35-40 min/day, 5 days a week over a period of 18 months in the Department of Physiology. Autonomic function testing was done in both the groups at zero time and after 18 months. We observed a statistically significant reduction in body mass index, waist circumference, systolic and diastolic blood pressure, and heart rate $(P<0.05)$, i.e. a significant positive effect was observed when yoga therapy was used as an adjunct in patients with coronary artery disease.

Effet d'une intervention basée sur le yoga sur le système nerveux autonome de patients atteints d'une maladie coronarienne : étude clinique randomisée et contrôlée

RÉSUMÉ Dans la présente étude, 258 patients consultant le service de cardiologie du centre hospitalier universitaire Chhatrapati Shahuji Maharaj de Lucknow (Inde) ont été sélectionnés pour cette intervention. Tous avaient reçu le diagnostic de maladie coronarienne. Les patients ont été répartis aléatoirement en deux groupes, le premier bénéficiant d'une intervention basée sur le yoga contrairement au second. Chaque groupe comptait 129 patients. Au total, 208 patients ont achevé le protocole de l'étude. L'intervention yogique durait 35 à 40 minutes par jour, 5 jours par semaine, sur une période de 18 mois dans le service de physiologie. Des tests de la fonction autonome ont été menés dans les deux groupes au début de l'étude et à 18 mois. Nous avons observé une réduction statistiquement significative de l'indice de masse corporelle, du tour de taille, de la pression systolique et diastolique, ainsi que de la fréquence cardiaque $(P<0,05)$. Un effet positif important a donc été observé lorsqu'une thérapie yogique a été appliquée en tant que traitement adjuvant chez des patients atteints d'une maladie coronarienne.

${ }^{7}$ Department of Physiology; ${ }^{2}$ Department of Cardiology; ${ }^{4}$ Department of Obstetrics and Gynaecology, Chhatrapati Shahuji Maharaj Medical University, Lucknow, Uttar Pradesh, India (Correspondence to N. Srivastava: drneenasrivastava@rediffmail.com).

${ }^{3}$ Department of Statistics, Lucknow University, Lucknow, Uttar Pradesh, India.

Received: 01/12/10; accepted: 01/11/11 


\section{Introduction}

Indians have considerably higher prevalence of premature coronary artery disease (CAD) and standardized mortality rates for $\mathrm{CAD}$ compared with Europeans, Chinese and Malays [1-4]. Projections on mortality rates due to $\mathrm{CAD}$ in India indicate almost 100\% increase from 1985 to 2015 [5]. The Indian population is more prone to $\mathrm{CAD}$ at a younger age: Indians are affected 5-10 years earlier than people in other communities $[6,7]$. This condition is the leading cause of morbidity and mortality in most industrialized societies [8].

It is believed that yogic practices were originated in the civilization of the Indian subcontinent. Yogic practices such as shatkarmas, asana, pranayama and meditation practices are very useful for physical, mental and spiritual development. Through these practices, individual consciousness is purified and the purified consciousness level can achieve salvation. Stress and anxiety levels can also be reduced through the practices of yoga. Relaxation asana like shavasana, makarasana, shashankasana are widely used for their stress releasing response.

The objective of this study was to determine the effect of yogic practices on sympathetic (blood pressure response to lying to standing test and handgrip test) and parasympathetic (30th: 15th ratio, E:I ratio, valsalva ratio) function in patients with $\mathrm{CAD}$.

\section{Methods}

The study was part of a larger study conducted under the Extra Mural Research Project of the Department of Department of Ayurveda, Yoga \& Naturopathy, Unani, Siddha and Homoeopathy (AYUSH), at the Indian Ministry of Health and Family Welfare. Some parts of the methodology have been described in a previously published study which was part of the same project [9].

Sample size was calculated as 200 This was increased to 258 to cover drop-outs, etc. Patients from the Department of Cardiology, Chhatrapati Shahuji Maharaj Medical University, Lucknow, India who fulfilled the inclusion criteria were invited to participate in the yoga interventional study. We approached 464 patients; 258 eligible patients agreed to participate (Figure 1). The study was carried out from February 2007 to July 2010. The progress of the participants through the study is described in Figure 1. The institutional Research Ethics Committee approved the study. All participants gave written informed consent to take part in the study before anthropometric measurements were taken.

A senior cardiologist referred the patients after examining their physical health and medication status. Patients were selected on the basis of

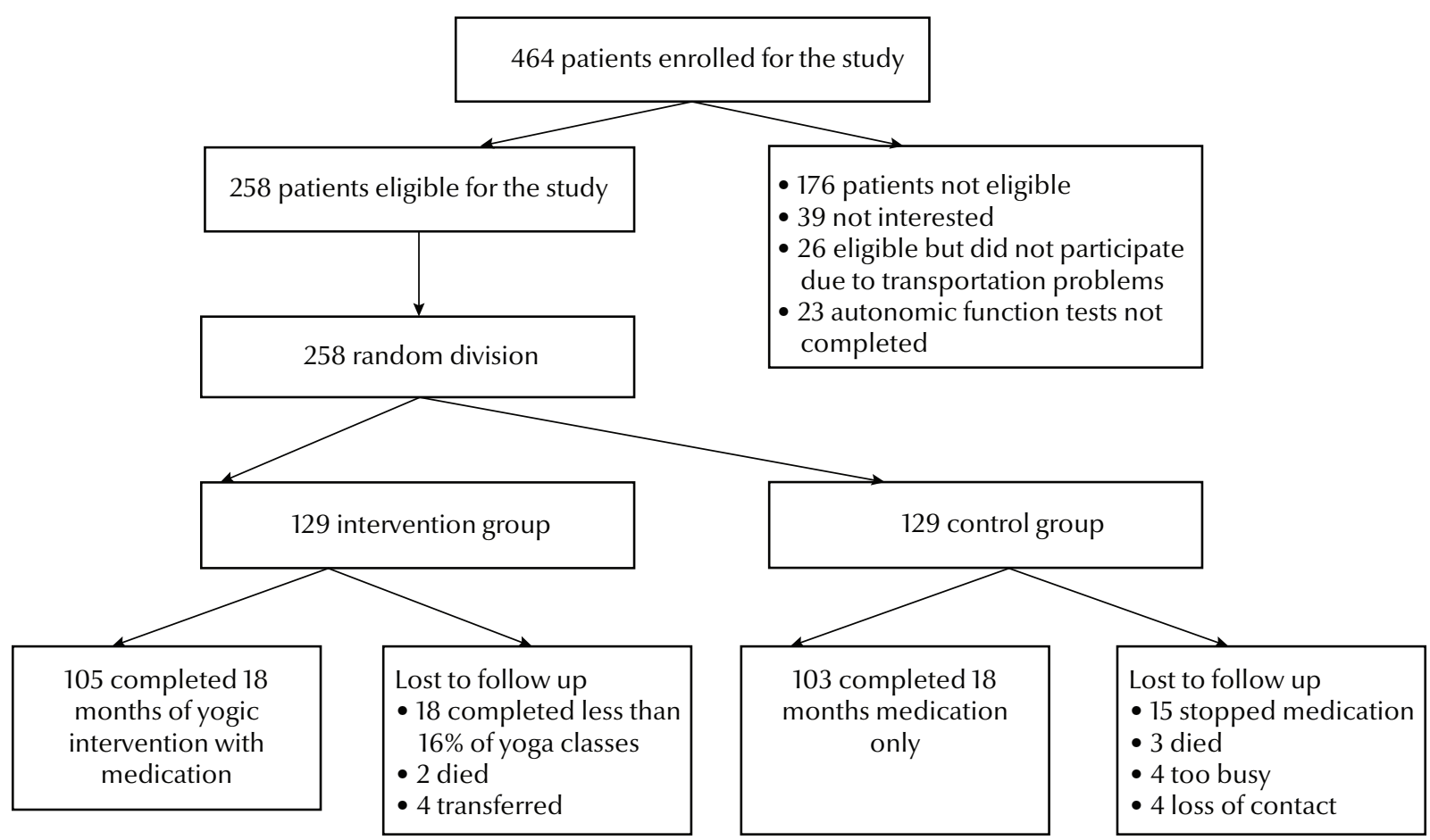

Figure 1 Flow-chart of the participants (patients with coronary artery disease, Lucknow, India) in the 18-month yogic intervention study showing progression of both intervention and control groups 
previous history and blood pressure level. Prescribed medications (metoprolol/atenolol, asprin, clopidogrel, atorvastatin/rosuvastatin, ramipril/ losartan/telmisartan) were continued in both groups.

Using a random number generator, each of the 258 patients selected was randomly assigned to one of 2 groups: (study group) medication + yoga, and (control group) medication only ( $n=129$ in each group). A professional not associated with this study generated the randomization scheme.

Yoga instructors (post graduate) with more than 2 years experience were selected to lead yogic practices. Yoga sessions were free of cost and all the necessary facilities were provided for the participants (airy room, yoga mats, jal neti pot, etc.).

Most of the participants were from Lucknow. Patients who dropped out of the study did not differ significantly in terms of age and sex; 208 completed the study protocol (Figure 1). Before the study all the participants were asked to maintain their routine activities and not initiate any new physical activities for the duration of the study.

Patients with proven CAD were recruited. Disease was diagnosed by history of electrocardiograms, echocardiography and treadmill testing. Their willingness to complete the entire span of the project ( 18 months) was assured. Patients who had other co-morbid conditions (e.g. malignant hypertension, diabetes mellitus, chronic obstructive pulmonary disease, asthma, diseases of the nervous system, endocrinal disorders, congenital heart disease) and patients with known complications of CAD, those on pacemakers, and those who had undergone bypass surgery were excluded from the study. Participants who did not participate in a minimum number of yoga intervention classes $(<60 \%)$ were not included in the final assessment (Figure 1).

\section{Autonomic functions tests}

A battery of 6 standard tests was used to assess the integrity of autonomic function status of the participants [10]. These tests were helpful in assessment of the reactivity of divisions of the autonomic nervous system, i.e. tests for parasympathetic and sympathetic function. The participants were made comfortable and the entire procedure was explained before the recording was done. They were asked to lie quietly on a couch and were connected to an electrocardiogram (ECG) monitor. Blood pressure was measured using a sphygmomanometer with a standard cuff around the left arm. After this preparation the participants were asked to remain supine and rest for about 10 minutes before the tests began. First the resting cardio-respiratory parameters (heart rate, and blood pressure) were recorded in a lying position.

\section{Test for parasympathetic reactivity}

Heart rate response to standing from the supine posture (30:15 ratio) [11]

For this test the heart rate was recorded in the lying posture by ECG; after recording a stable baseline resting heart rate, the patient was asked to get up unaided and the heart rate was continuously recorded. From the post-standing record, the ratio of the longest to the shortest R-R interval (approximately corresponding to the 30th and 15th beats, by convention: maximum $\mathrm{R}-\mathrm{R}$ interval around 30th beat, minimum $\mathrm{R}-\mathrm{R}$ interval around 15 th beat) was recorded.

\section{Slow deep breathing test}

After 3-5 min in a sitting position, the participants were asked to take deep, slow breaths at a rate of 6 breaths per minute [12] in the supine position for $1 \mathrm{~min}$ [11]. An automatic event marker was used to mark the inspiratory (I) and expiratory (E) phases, indicating delivery of signals. The E:I ratio was the ratio of averaged maximum and minimum $\mathrm{R}-\mathrm{R}$ intervals during expiration and inspiration respectively during the manoeuvre. We checked whether the respective phase of respiration coincided with the signal marking, which was confirmation of the precise execution of the test. If not, the test was repeated after adequate interval or at the end.

E:I ratio $=$ average maximum $\mathrm{R}-\mathrm{R}$ interval during expiration/average minimum R-R interval during inspiration.

\section{Valsalva manoeuvre [13]}

The valsalva manoeuvre comprises abrupt, transient and voluntary elevation of intra-thoracic and intraabdominal pressure provoked by straining [14], expiring forcefully through a mouthpiece attached to a manometer to generate a pressure of $40 \mathrm{mmHg}$ and maintaining this pressure for 15 seconds after which the participants released the effort and the mouthpiece was removed. Due care was taken to prevent deep breathing before and after the release of strain. The participants were asked to sit quietly and breathe normally after the test.

Valsalva ratio $=$ longest $\mathrm{R}-\mathrm{R}$ interval after manoeuvre (phase IV)/shortest $\mathrm{R}-\mathrm{R}$ interval during manoeuvre (phase II).

\section{Tests for sympathetic reactivity}

\section{Blood pressure response to lying to standing test}

The participants were asked to stand up quickly in 3 seconds and remain standing quietly for 2 minutes putting equal weight on each leg and without any support [15]. Blood pressure was recorded at $0 \mathrm{~min}$ (baseline; in lying position) and 0.5, 1.0 and 2.0 min while standing up. The differences in systolic and diastolic pressure from baseline were calculated. The participants were asked to sit quietly after the test.

\section{Hand grip test [10]}

A very light, small, hand-held handgrip dynamometer, which shows isometric strength, was used for this 
test. After instruction in the use of the dynamometer, baseline blood pressure was recorded. The participants were then asked to grip the dynamometer with their dominant hand maximally. This was repeated 3 times at short intervals. The maximum of these 3 readings was taken as maximum voluntary contraction. For the test, the subjects were asked to maintain the pressure on the dynamometer for $5 \mathrm{~min}$ at $30 \%$ of maximum voluntary contraction. Blood pressure was recorded on the non-exercising arm at $1 \mathrm{~min}$ and $2 \mathrm{~min}$ after the onset of isometric contraction (before release of grip). The changes in systolic and diastolic blood pressure were measured.

\section{Yogic intervention}

Under the guidance and supervision of yoga experts, participants in the yoga group performed yoga practices as directed. These practices are described in detail in a previously published study which was part of the same project [9].

The total duration of these practices was 35-40 min/day, 5 days a week over the 18 months of the study in the Department of Physiology at Chhatrapati Sahuji Maharaj Medical University.

Using the independent $t$-test of differences $[16,17]$ to analyse the differences between baseline values and post-intervention (after 18 months) values for each variable in both groups (yoga and non-yoga). $P<0.05$ was considered statistically significant,

\section{Results}

The baseline characteristics for both groups are presented in Table 1. Patients of both sexes were included and the overall age range was 35-82 years. In the control group mean age was 56.4 [standard deviation (SD) 10.9] years and in the yoga group mean age was 59.1 (SD 9.9) years.

After 18 months 208 participants remained in the study, 105 in the

\begin{tabular}{|c|c|c|}
\hline \multicolumn{3}{|c|}{$\begin{array}{l}\text { Table } 1 \text { Baseline characteristics of patients with coronary artery disease who } \\
\text { participated in the yoga intervention study, Lucknow, India, 2007-2009 }\end{array}$} \\
\hline Characteristic & $\begin{array}{l}\text { Control group }{ }^{\mathrm{a}} \\
(n=129)\end{array}$ & $\begin{array}{l}\text { Yogic group } \\
(n=129)\end{array}$ \\
\hline & Mean (SD) & Mean (SD) \\
\hline Age (years) & $56.4(10.9)$ & $59.1(9.9)$ \\
\hline Height $(\mathrm{cm})$ & $161.6(8.4)$ & $161.5(9.0)$ \\
\hline Weight (kg) & $65.6(12.3)$ & $64.9(9.3)$ \\
\hline WC $(\mathrm{cm})$ & $94.2(10.5)$ & $93.0(8.3)$ \\
\hline $\mathrm{HC}(\mathrm{cm})$ & $95.6(11.3)$ & $94.5(9.4)$ \\
\hline $\mathrm{SBP}(\mathrm{mmHg})$ & $129.8(14.1)$ & $130.1(10.3)$ \\
\hline $\mathrm{DBP}(\mathrm{mmHg})$ & $82.3(8.6)$ & $83.7(8.0)$ \\
\hline Heart rate (beats/min) & 71.6 (11.4) & $73.0(9.1$ \\
\hline
\end{tabular}

${ }^{a} 104$ males, 25 females.

${ }^{6} 703$ males, 26 females.

$S D=$ standard deviation; $W C=$ waist circumference, $H C=$ hip circumference, $S B P=$ systolic blood pressure; $D B P=$ diastolic blood pressure.

intervention group and 103 in the control group: 50 had been lost to followup, 26 in the control group and 24 in the intervention group (Figure 1).

In the intervention group mean body mass index (BMI) decreased to $24.3(\mathrm{SD} 3.5) \mathrm{kg} / \mathrm{m}^{2}$ from the baseline of $24.8(\mathrm{SD} 3.8) \mathrm{kg} / \mathrm{m}^{2}$, whereas in the control group mean BMI remained substantially the same (Table 2). Mean systolic blood pressure (SBP) fell to 123.1 (SD 9.4) $\mathrm{mmHg}$ from the baseline level of 130.1 (SD 10.3) mmHg and in the control group mean SBP fell slightly to 129.1 (SD 9.3) $\mathrm{mmHg}$ from the baseline value of 129.8 (SD 14.1) $\mathrm{mmHg}$. The DBP level in the intervention group fell to 80.48 (SD 5.12) $\mathrm{mmHg}$ from the baseline level of 83.7 (SD 8.0) $\mathrm{mmHg}$ and in control group mean diastolic blood pressure (DBP) rose to 83.8 (SD 5.7) $\mathrm{mmHg}$ from the baseline level of 82.3 (SD 8.6) $\mathrm{mmHg}$.

There were significant changes observed in BMI $(P<0.0001)$, waist:hip ratio $(P<0.005)$, SBP $(P<0.002)$, DBP $(P<0.0002)$ and heart rate $(P<0.0006)$ (Table 3).

\section{Discussion}

High blood pressure is a major cause of cardiovascular disease and primary hypertension is a frequent pathological condition. Sympathetic hyperactivity may be involved in primary hypertension. Hypertensive patients have been found to have a significantly higher sympathetic response to central and peripheral stimulations and a significantly lower parasympathetic response when compared to normotensive controls [18]. In our study, BMI, waist:hip ratio, SBP, DBP and resting heart rate decreased significantly more in the intervention group than in the control group. In a study in Bombay, the shavasana group had a significant decrease in blood pressure [19]. Yogic practices have been shown to have significant benefits for cardiovascular health: through these practices heart rate, systolic blood pressure and diastolic blood pressure decrease significantly [20-22]. In another study it was observed that after fast pranayama basal heart rate was reduced [23]. A similar study conducted in Puducherry, India and it was found that after yogic practices heart rate was reduced significantly in hypertensive patients [24].

In our study resting heart rate decreased significantly in the yoga intervention group but not in the control group. Vempati and Telles reported that the rate of respiration and heart 


\begin{tabular}{|c|c|c|c|c|}
\hline \multirow[t]{3}{*}{ Characteristic } & \multicolumn{2}{|c|}{ Control group } & \multicolumn{2}{|c|}{ Yogic group } \\
\hline & $\begin{array}{c}\text { Pre } \\
(n=129)\end{array}$ & $\begin{array}{c}\text { Post } \\
(n=103)\end{array}$ & $\begin{array}{c}\text { Pre } \\
(n=129)\end{array}$ & $\begin{array}{c}\text { Post } \\
(n=105)\end{array}$ \\
\hline & Mean (SD) & Mean (SD) & Mean (SD) & Mean (SD) \\
\hline $\mathrm{BMI}\left(\mathrm{kg} / \mathrm{m}^{2}\right)$ & $25.1(4.8)$ & $25.1(4.6)$ & $24.8(3.8)$ & $24.3(3.5)$ \\
\hline Waist:hip ratio & $1.0(0.1)$ & $1.0(0.1)$ & $1.0(0.1)$ & $1.0(0.1)$ \\
\hline $\mathrm{SBP}(\mathrm{mmHg})$ & $129.8(14.1)$ & $129.1(9.3)$ & $130.1(10.3)$ & $123.1(9.4)$ \\
\hline $\mathrm{DBP}(\mathrm{mmHg})$ & $82.3(8.6)$ & $83.8(5.7)$ & $83.7(8.0)$ & $80.5(5.1)$ \\
\hline Heart rate (beats/min) & $71.6(11.4)$ & $73.3(8.7)$ & $73.0(9.1$ & $70.5(7.5)$ \\
\hline Fall in SBP $(\mathrm{mmHg})^{a}$ & $10.3(4.3)$ & $9.3(4.2)$ & $10.5(4.4)$ & $10.1(4.3)$ \\
\hline $\begin{array}{l}\text { Increase in DBP after } \\
\text { sustained hand grip }(\mathrm{mmHg})\end{array}$ & $10.6(6.1)$ & $10.5(5.6)$ & $12.6(4.8)$ & $12.1(4.7)$ \\
\hline Expiration-inspiration ratio & $1.2(0.3)$ & $1.1(0.2)$ & $1.1(0.2)$ & $1.2(0.1))$ \\
\hline $30: 15$ beat ratio & $1.1(0.2)$ & $1.1(0.2)$ & $1.1(0.3)$ & $1.1(0.2)$ \\
\hline Valsalva ratio & $1.6(0.8)$ & $1.5(0.6)$ & $1.5(0.7)$ & $1.6(0.9)$ \\
\hline $\mathrm{LF} / \mathrm{HF}$ ratio & $1.6(1.3)$ & $1.6(1.2)$ & $1.7(1.3)$ & $1.6(1.2)$ \\
\hline
\end{tabular}

${ }^{a}$ Differences in systolic blood pressure from the baseline (lying position) and after 2 minutes while standing.

$S D=$ standard deviation; $B M I=$ body mass index; $S B P=$ systolic blood pressure; $D B P=$ diastolic blood pressure; $L F=l o w$ frequency; $H F=$ high frequency.

rate decreased significantly after yogabased relaxation [25]. There was no significant change in the heart rate in the control group in our study. The results indicate that sympathetic reactivity reduced to some extent in yoga group, but not statistically significantly. This is in contrast with the findings of the study of Mourya et al. [26].
Yogic practices can affect very short term to body mass index and blood pressure levels. Body mass index and waist hip ratio are the physiological parameters for measuring obesity. Both the variables decreased significantly when compared with control. Both the studies got similar results after short term yogic intervention $[27,28]$. There is also evidence that om chanting effects changes in various biochemical levels such as endorphins, cortisol, adrenocorticotrophic hormone, interleukin-1, and immunoglobulin $\mathrm{A}$, all of which affect the immune system. In a study in St Petersburg it was found that in subjects who adopted the cobra posture (bhujangasana), the production of steroid hormones was controlled by neural mechanisms [29].

\begin{tabular}{|c|c|c|c|}
\hline \multirow[t]{3}{*}{ Characteristic } & \multicolumn{2}{|c|}{ Difference from baseline } & \multirow[t]{3}{*}{$P$-value } \\
\hline & Control group & Yogic group & \\
\hline & Mean (SD) & Mean (SD) & \\
\hline $\mathrm{BMI}\left(\mathrm{kg} / \mathrm{m}^{2}\right)$ & $-0.03(1.07)$ & $0.52(0.89)$ & $0.0001^{* *}$ \\
\hline Waist:hip ratio & $-0.004(0.09)$ & $0.02(0.03)$ & $0.005^{* *}$ \\
\hline Heart rate (beats/min) & $-1.78(9.13)$ & $2.58(8.79)$ & $0.0006^{* *}$ \\
\hline $\mathrm{SBP}(\mathrm{mmHg})$ & $0.70(10.81)$ & $5.41(11.40)$ & $0.002^{* *}$ \\
\hline $\mathrm{DBP}(\mathrm{mmHg})$ & $-1.51(8.69)$ & $3.27(8.01)$ & $0.0002^{* *}$ \\
\hline Fall in SBP (mmHg) & $0.98(4.76)$ & $0.36(5.28)$ & 0.38 \\
\hline Sustained hand grip DBP $(\mathrm{mmHg})$ & $0.09(4.57)$ & $0.43(4.55)$ & 0.59 \\
\hline Valsalva ratio & $0.07(0.60)$ & $0.13(0.07)$ & 0.44 \\
\hline Expiration-inspiration ratio & $0.01(0.31)$ & $0.06(0.29)$ & 0.88 \\
\hline Standing ratio & $0.03(0.22)$ & $0.05(0.26)$ & 0.49 \\
\hline $\mathrm{LF} / \mathrm{HF}$ ratio & $0.01(1.18)$ & $0.18(1.12)$ & 0.27 \\
\hline
\end{tabular}

**Statistically significant $(P<0.01)$.

$S D=$ standard deviation; $B M I=$ body mass index; $S B P=$ systolic blood pressure; $D B P=$ diastolic blood pressure; $L F=$ low frequency; $H F=$ high frequency. 


\section{Limitations of the study}

The limitations of this study include the fact that our study group was taken from only the urban population (the study was conducted in a medical university situated in urban area so it was not possible for members of the rural population to attend a yoga class of such long duration in the mornings). Parameters tested in this study were indirectly related to $\mathrm{CAD}$; there were no parameters directly related. Measuring cardiac output, ECG monitoring, etc., however, did provide accurate information related to $\mathrm{CAD}$.
The diet factor plays a vital role in yogic interventional studies. However, in this study we did not take into consideration the effect of diet. Measuring the effects of diet is very complicated in outpatients; it is only possible in inpatients. In this study we requested that patients maintain their normal daily routine.

Qualitative analysis of the participants was also useful for discussing the parameters which changed after yogic intervention. This study may provide a base for clinicians and policy-makers indicating that yogic intervention could be prescribed as an adjunct to medical treatment.

\section{Acknowledgements}

We are very grateful to the entire yoga team for its coordination. We are also grateful to all the staff of the Departments of Physiology, Pathology and Cardiology at Chhatrapati Shahuji Maharaj Medical University, Lucknow, for their cooperation during the entire study.

Funding: This study received the financial support of the Department of AYUSH, Ministry of Health and Family Welfare, Government of India.

Competing interests: None declared.

\section{References}

1. Enas EA et al. Coronary heart disease and its risk factors in firstgeneration immigrant Asian Indians to the United States of America. Indian Heart Journal, 1996, 48:343-353.

2. Hughes $\mathrm{K}$ et al. Ischemic heart disease and its risk factors in Singapore in comparison with other countries. Annals of the Academy of Medicine Singapore, 1989, 18:245-249.

3. Anand SS et al. Differences in risk factors, atherosclerosis, and cardiovascular disease between ethnic groups in Canada: the Study of Health Assessment and Risk in Ethnic groups (SHARE). Lancet, 2000, 356:279-284.

4. Balarajan R. Ethnic differences in mortality from ischaemic heart disease and cerebrovascular disease in England and Wales. BMJ (Clinical Research Ed.), 1991, 302:560-564.

5. Bulato RA, Stephens PW. Global estimates and projections of mortality by cause, 1970-2015. Washington DC, World Bank, Population Health and Nutrition Department, 1992:100 (Preworking paper 1007).

6. Janus ED et al. Council on Arteriosclerosis of the International Society and Federation of Cardiology. The modernization of Asia. Implications for coronary heart disease. Circulation, 1996, 94:2671-2673.

7. McKeigue PM et al. Association of early-onset coronary heart disease in South Asian men with glucose intolerance and hyperinsulinemia. Circulation, 1993, 87:152-161.

8. Enas EA, Senthilkumar A. Coronary artery disease in Asian Indians: an update and review. The Internet Journal of Cardiology. 2001, 1(2).

9. Pal A et al. Effect of yogic practices on lipid profile and body fat composition in patients of coronary artery disease. Complementary Therapies in Medicine, 2011, 19(3):122-127 (http:// www.researchgate.net/publication/51191805_Effect_of_yogic_practices_on_lipid_profile_and_body_fat_composition_ in_patients_of_coronary_artery_disease, accessed 21 February 2013).

10. Ewing RJ. In: Bannister R, Mathias CJ, eds. Autonomic failure: $a$ text book of clinical disorders of the autonomic nervous system. Oxford, Oxford University Press, 1992:312-333.

11. Weiling $\mathrm{W}$ et al. Testing for autonomic neuropathy: initial heart rate response to active and passive change of posture. Clinical Physiology, 1985, 5(Suppl. 5):23-27.

12. Sundkvist G, Almér L, Lilja B. Respiratory influence on heart rate in diabetes mellitus. British Medical Journal, 1979, 1:924-925.
13. Levin AB. A simple test of cardiac function based upon the heart rate changes induced by the Valsalva maneuver. American Journal of Cardiology, 1966, 18:90-99.

14. Korner PI, Tonkin AM, Uther JB. Reflex and mechanical circulatory effects of graded Valsalva maneuvers in normal man. Journal of Applied Physiology, 1976, 40:434-440.

15. Weiling W, Borst C, Karemaker JM. Testing for autonomic neuropathy: Initial heart rate response to active and passive change of posture. Clinical Physiology, 1985, 23: 678-690.

16. Guyatt G, Walter S, Norman G. Measuring change over time: assessing the usefulness of evaluative instruments. Journal of Chronic Diseases, 1987, 40:171-178.

17. MacKenzie CR et al. Can the sickness impact profile measure change? An example of scale assessment. Journal of Chronic Diseases, 1986, 39:429-438.

18. Aboudrar $\mathrm{S}$ et al. Les réponses sympathiques dans I'hypertension artérielle essentielle [Sympathetic response in primary hypertension]. Annales de Cardiologie et d'Angéiologie, 2009, 58(3):139-143.

19. Sundar $\mathrm{S}$ et al. Role of yoga in management of essential hypertension. Acta Cardiologica, 1984, 39(3):203-208.

20. Lakkireddy D et al. Effect of yoga on arrhythmia burden, anxiety, depression, and quality of life in paroxysmal atrial fibrillation: the YOGA My Heart Study. Journal of the American College of Cardiology, 2013, (doi: 10.1016/j.jacc.2012.11.060).

21. Monika et al. Effect of yoga nidra on physiological variables in patients of menstrual disturbances of reproductive age. Indian Journal of Physiology and Pharmacology, 2012, 56(2):161-167.

22. Miles SC et al. Arterial blood pressure and cardiovascular responses to yoga practice. Alternative Therapies in Health and Medicine, 2013, 19(1):38-45.

23. Veerabhadrappa SG et al. Effect of yogic bellows on cardiovascular autonomic reactivity. Journal of Cardiovascular Disease Research. 2011, 2(4):223-227.

24. Bhavanani AB, Sanjay Z, Madanmohan. Immediate effect of sukha pranayama on cardiovascular variables in patients of hypertension. International Journal of Yoga Therapy, 2011, (21):73-76.

25. Vempati RP, Telles S. Yoga-based guided relaxation reduces sympathetic activity judged from baseline levels. Psychological Reports, 2002, 90:487-494. 
26. Mourya M et al. Effect of slow- and fast-breathing exercises on autonomic functions in patients with essential hypertension. Journal of Alternative \& Complementary Medicine, 2009, 15(7):711-717.

27. Sarvottam K et al. Adiponectin, interleukin-6, and cardiovascular disease risk factors are modified by a short-term yoga-based lifestyle intervention in overweight and obese men, Journal of Alternative \& Complementary Medicine, 2012 (Dec 4, Epub ahead of print).

28. Seo DY et al, Yoga training improves metabolic parameters in obese boys. Korean Journal of Physiology \& Pharmacology, 2012, 16(3):175-180.

29. Ernst E. Breathing techniques-adjunctive treatment modalities for asthma? European Respiratory Journal, 2000, 5:969-972.

\section{Global atlas on cardiovascular disease prevention and control: policies, strategies and interventions}

As the magnitude of cardiovascular diseases (CVDs) continues to accelerate globally, the pressing need for increased awareness and for stronger and more focused international and country responses is increasingly recognized. The abovementioned atlas on cardiovascular disease prevention and control is part of the response to this need.

It documents the magnitude of the problem, using global cardiovascular mortality and morbidity data. It demonstrates the inequities in access to protection, exposure to risk, and access to care as the cause of major inequalities between countries and populations in the occurrence and outcome of CVDs. The report has graphs showing mortality rates of CVDs by age, by country/region, and is divided into three main sections: Section A: Cardiovascular diseases due to atherosclerosis; Section B: Other cardiovascular diseases; and Section C: Prevention and control of CVDs - Policies, strategies and interventions.

This publication can be found at: http://www.who.int/cardiovascular_diseases/publications/atlas_cvd/en/index. html 